\title{
Medidas de adesão entre agregado e ligante asfáltico
}

\author{
Kamilla Lima Vasconcelos ${ }^{1}$; Amit Bhasin²; Dallas N. Little ${ }^{3}$; Jorge Barbosa Soares ${ }^{4}$
}

\begin{abstract}
Resumo: A adesão entre agregado e ligante asfáltico vem sendo apontada como uma propriedade de grande importância para a causa de defeitos encontrados em pavimentos asfálticos, tais como trincas por fadiga, e dano por umidade. Diferentes mecanismos existem na literatura para explicar a adesão entre os dois materiais, porém, estes mecanismos podem ser resumidos em três grandes grupos: intertravamento mecânico, adesão física, e interação química. A ocorrência de mais de um mecanismo simultaneamente parece ser o fenômeno mais provável, sendo a relevância de cada um dependente das características físicas e químicas do agregado e do ligante asfáltico. No presente trabalho, dois procedimentos foram utilizados para acessar a adesão entre agregado e ligante. O primeiro constou do cálculo do trabalho de adesão através da energia livre de superfície dos materiais envolvidos e o segundo, da determinação da entalpia de imersão quando soluções de asfalto são postas em contato com o agregado. Todos os materiais usados foram provenientes da biblioteca de referência do SHRP sendo um pedregulho como agregado, e três diferentes ligantes asfálticos. Os resultados mostraram a capacidade do microcalorímetro em detectar possíveis interações químicas na adesão entre agregado e ligante asfáltico, em conjunto com adesão física. A presença de grupos funcionais mais fortemente adsorvidos pela superfície dos agregados justificou os maiores valores de entalpia de imersão para as combinações onde esses grupos se encontravam presentes.
\end{abstract}

\begin{abstract}
Adhesion between the asphalt binder and the aggregate is critical to the performance and durability of asphalt mixtures. According to the literature, distresses mechanisms such as fatigue cracking and moisture induced damage are correlated to the nature and quality of adhesion between these two materials. Different mechanisms already exist to explain adhesion, but they can be summarized in three main groups: mechanical interlocking, physical adhesion and chemical interaction. Although discussions of isolated theories and mechanisms help to clarify the understanding of adhesion, they can rarely be separated completely to each other. The physical and chemical characteristics of asphalt and aggregate will in fact determine the relevance of each of these mechanisms. This study presents the results of two procedures for adhesion measurement: (i) an indirect method based on the surface free energy components of asphalt binder and aggregate; and (ii) the determination of the enthalpy of immersion through the use of a microcalorimeter. The materials used, a gravel as aggregate and three different neat asphalts, were provided by the Strategic Highway Research Program's Material Reference Library. The results showed the capacity of the microcalorimeter in detecting possible chemical reactions together with physical adhesion. The presence of functional groups more strongly adsorbed by the aggregate surface justified the higher values of the heat of immersion.
\end{abstract}

\section{INTRODUÇÃO}

A adesão entre agregado e ligante asfáltico é fator de grande relevância para a durabilidade e o desempenho dos pavimentos. Conforme apontado pela literatura, defeitos como deformação permanente, fadiga e dano por umidade estão diretamente relacionados com a natureza e qualidade da adesão entre os dois materiais (Plancher et al., 1977; Petersen et al., 1982; Curtis et al., 1991; Cheng et al., 2002a).

Pesquisas desenvolvidas na Texas A\&M University

\footnotetext{
${ }^{1}$ Kamilla Lima Vasconcelos, Materials and Pavement Division, Texas Transportation Institute, Texas A\&M University. College Station, Texas, EUA (e-mail: kamilla@ttimail.tau.edu).

${ }^{2}$ Amit Bhasin, Materials and Pavement Division, Texas Transportation Institute, Texas A\&M University. College Station, Texas, EUA (e-mail: a-bhasin@ttimail.tau.edu).

${ }^{3}$ Dallas N. Little, Materials and Pavement Division, Texas Transportation Institute, Texas A\&M University. College Station, Texas, EUA (e-mail: d-little@ttimail.tau.edu).

${ }^{4}$ Jorge Barbosa Soares, Laboratório de Mecânica dos Pavimentos, Departamento de Engenharia de Transportes, Universidade Federal do Ceará (e-mail: jsoares@det.ufc.br).
}

Manuscrito recebido em 6/7/2007 e aprovado para publicação em 10/12/2007. Este artigo é parte de TRANSPORTES, volume XVI, número 1, de junho de 2008. ISSN: 1415-7713. apontam para uma correlação entre a espessura do filme de ligante e o tipo de falha (adesiva ou coesiva) em misturas asfálticas. Filmes finos de ligante asfáltico estão normalmente associados com falhas adesivas, enquanto filmes mais espessos caracterizam falhas coesivas (Lytton, 2004). Um estudo pioneiro realizado por Marek e Herrin (1958) já apontavam para essa correlação. Os autores observaram três diferentes tipos de falha: (i) por "quebra", (ii) por "fluência", e (iii) intermediária, a depender das condições de ensaio (espessura do filme de ligante, taxa de deformação, consistência do ligante, etc). Masad et al. (2006) demonstraram o uso da resistência de ligação por adesão (adhesive bond strength) entre agregado e ligante asfáltico na modelagem de características de trincas por fadiga em misturas de mástique.

A adesão entre agregado e ligante é normalmente caracterizada por meio de intertravamento mecânico, adesão física, e interação química, podendo haver a existência dos três processos simultaneamente. Apesar dos mecanismos que controlam a adesão já estarem estabelecidos em literatura, ainda existe uma carência na utilização destes na seleção de materiais durante o projeto de misturas asfálticas duráveis. 
$\mathrm{O}$ presente trabalho utilizou materiais provenientes da biblioteca de referência do SHRP para caracterização da adesão entre agregado e ligante asfáltico. Dois processos foram utilizados: (i) medida indireta através da energia livre de superfície dos materiais; e (ii) medida da entalpia de imersão pela utilização de um microcalorímetro. Estes encontram-se descritos nos ítens a seguir.

\section{ADESÃO ENTRE AGREGADO E LIGANTE ASFÁLTICO}

O estudo da adesão entre agregado e ligante asfáltico vem sendo abordado de forma crescente, devido a grande correlação que vem sendo apontada entre esta e os diversos defeitos existentes em pavimentos asfáticos.

No estudo realizado por Hefer et al. (2005), os autores apresentaram em forma de revisão bibliográfica cinco teorias/mecanismos para o fenômeno da adesão entre agregado e ligante: (i) teoria da camada mais fraca, (ii) teoria mecânica, (iii) teoria eletrostática, (iv) teoria da ligação química, e (v) teoria da termodinâmica.

A teoria da camada mais fraca atribui a falha por adesão à existência de uma região entre "adesivo" e "substrato" de menor resistência à falha por coesão. Essa deficiência pode ser atribuída à presença de contaminantes no agregado (Tarrar e Wagh, 1992), ou mesmo a sua alta porosidade (Jeon e Curtis, 1990). Nesse último caso, as frações de asfalto de menor peso molecular são absorvidas preferencialmente por esses poros, fazendo com que as frações remanescentes sejam mais rígidas, e conseqüentemente mais susceptíveis a trincas.

A teoria mecânica é a mais comumente aceita, principalmente por se tratar de um mecanismo intuitivo e macroscópico, onde o ligante asfáltico adere firmemente ao agregado, mesmo não havendo nenhum tipo de interação química, ou formação de camadas diferenciadas (Allen, 1992). A estrutura física da superficie do agregado é de importância chave para esse mecanismo, de forma que agregados rugosos conseqüentemente apresentarão melhor adesão do que agregados polidos (Dessouky et al., 2006).

A teoria eletrostática assume que agregado e ligante asfáltico estão ligados devido a presença de cargas em sua interface, acarretando na formação de dipolos. No estudo realizado por Robert (1977), porém, conclui-se que menos de $10 \%$ do trabalho da adesão da borracha com diferentes materiais é devido à interação eletrostática.

A teoria da ligação química talvez seja a mais complexa, visto a alta variabilidade existente nas composições dos agregados e ligantes utilizados em misturas asfálticas. Os grupos funcionais presentes no ligante asfáltico e no agregado são o fator mais importante de acordo com essa teoria. No estudo realizado por Plancher et al. (1977) e Petersen et al. (1982), os autores apresentam os grupos mais fortemente adsorvidos pela superfície dos agregados, além da susceptibilidade desses grupos à presença de água. De forma interessante observou-se que grupos funcionais como: ácidos carboxílicos, anidridos, e 2-quinolone (Figura 1) são os mais fortemente adsorvidos, porém também os de maior susceptibilidade à ação da água. A cal hidratada é comumente utilizada como melhorador dessa susceptibilidade em misturas asfálticas e no estudo desenvolvido por Little e Petersen (2005), os autores apontam a efetividade da cal hidratada como dependente da interação fisico-química da mesma com o ligante asfáltico utilizado. Nos casos mais efetivos, a cal hidratada reage com grupos funcionais do tipo ácido carboxílico formando sais de cálcio, que são reconhecidos como sais insolúveis.

A teoria da termodinâmica baseia-se na adesão entre agregado e ligante asfáltico através de forças intermoleculares na interface. A magnitude dessas forças está geralmente ligada a propriedades termodinâmicas, como é o caso da energia livre de superfície (surface free energy), $\gamma$, dos materiais envolvidos no processo. A definição de energia livre de superfície através da termodinâmica é o trabalho reversível necessário para criar uma unidade de área de uma nova superfície. Conforme mencionado por Bhasin (2006), os termos: tensão de superfície, energia de superfície, e energia livre de superfície são usados permutavelmente, apesar de 'energia livre de superfície' ser o termo correto para utilizacação nos princípios da termodinâmica.

\subsection{Energia livre de superfície}

Diferentes teorias explicam a origem molecular da energia livre de superfície em sólidos. A teoria de Good-van Oss-Choudhury (GVOC), também conhecida

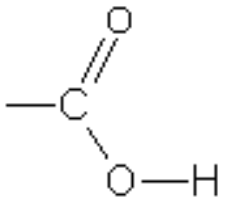

(a)

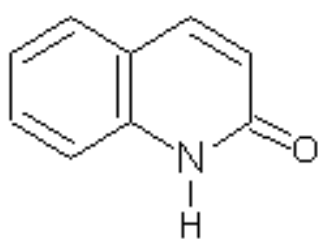

(b)

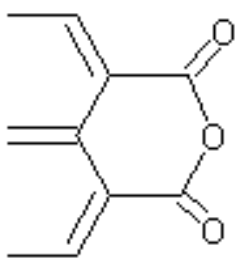

(c)

Figura 1: Exemplos de grupos funcionais químicos presentes no ligante asfáltico: (a) ácido carboxílico, (b) 2-quinolone, e (c) anidrido 
como teoria ácido-base, é amplamente utilizada para explicar os componentes da energia livre de superfície em diversos materiais (Van Oss et al., 1988; Van Oss, 1994). De acordo com essa teoria, a total energia livre de superfície de qualquer material é dividida em três componentes, conforme o tipo de força molecular em sua superfície. Esses componentes são: (i) componente não-polar, também conhecido como Lifshitz-van de Waals, ou componente dispersivo; (ii) componente Lewis ácido; e (iii) componente Lewis básico. A total energia livre de superfície é obtida através da combinação dos três componentes através da Equação 1:

$$
\gamma=\gamma^{L W}+\gamma^{+-}=\gamma^{L W}+2 \sqrt{\gamma^{+} \gamma^{-}}
$$

$$
\begin{array}{ll}
\begin{array}{ll}
\text { em que, } \\
\gamma
\end{array} & \text { é a total energia livre de superfície, } \\
\gamma^{L W} & \text { o componente Lifshitz-van der Waals, ou disper- } \\
& \text { sivo, } \\
\gamma^{+-} & \text {o componente ácido-base, } \\
\gamma^{+} & \text {o componente Lewis ácido, e } \\
\gamma^{-} & \text {o componente Lewis básico. }
\end{array}
$$

Essa teoria também é aplicada para a determinação do trabalho de adesão, $W_{A B}$, entre dois materiais A e $\mathrm{B}$ em termos dos componentes de suas energias livre de superfície através da Equação 2:

$$
W_{A B}=2 \sqrt{\gamma_{A}^{L W} \gamma_{B}^{L W}}+2 \sqrt{\gamma_{A}^{+} \gamma_{B}^{-}}+2 \sqrt{\gamma_{A}^{-} \gamma_{B}^{+}}
$$

Diferentes métodos estão disponíveis para medidas da energia livre de superfície de sólidos e líquidos. Cheng (2002b) e Bhasin (2006) recomendam a utilização do Wilhelmy Plate (WP) para ligantes asfálticos, e do Universal Sorption Device (USD) para agregados. O WP baseia-se na medida de ângulos de contato, e o USD na adsorção de vapor, conforme ilustrado nas Figuras 2 e 3. Estes foram os testes utilizados para determinação da energia livre de superfície dos ligantes asfálticos e do agregado no presente estudo. Maiores informações sobre a execução dos mesmos podem ser obtidas em Bhasin (2006) e Hefer et al. (2006).

\section{MICROCALORÍMETRO}

O microcalorímetro é um equipamento utilizado para medir a energia gerada durante o processo de uma reação química, mudança de estado, formação de uma solução, etc. Ensley et al. (1984) utilizaram o microcalorímetro para determinar a temperatura 'ótima' de adesão entre agregado e ligante, de forma que haja dissociação do ligante, redução na viscosidade, porém a temperatura não é elevada a ponto de causar o desprendimento (desorption) entre os dois materiais. No estudo desenvolvido por Bhasin (2006), o autor utili-

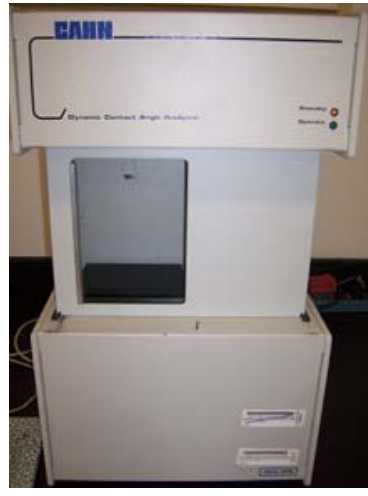

(a)

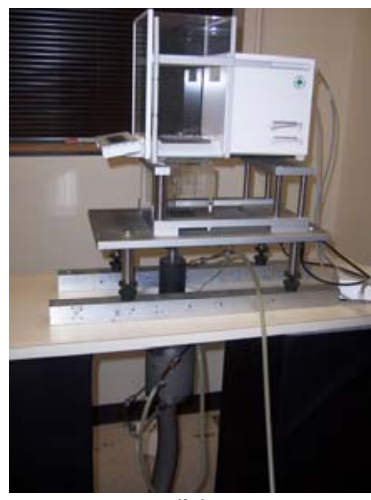

(b)
Figura 2: (a) Wilhelmy Plate, e (b) Universal Sorption Device

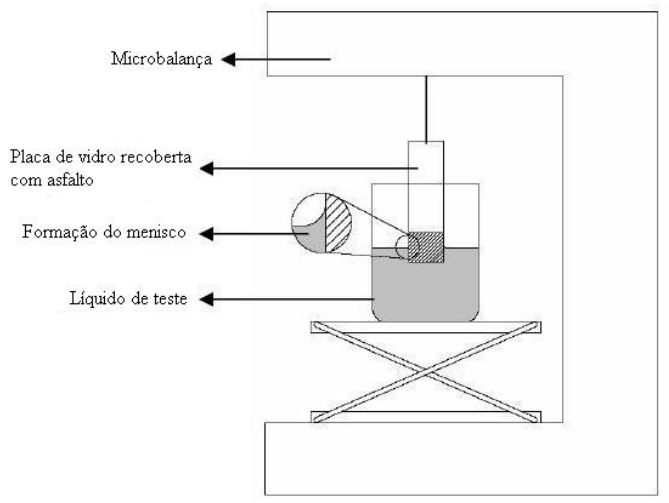

(a)

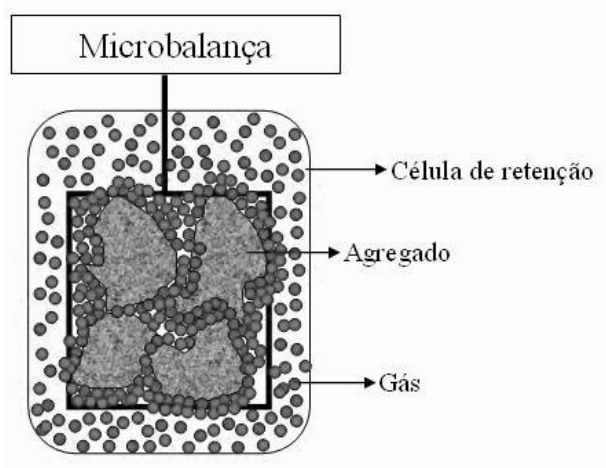

(b)

Figura 3: Desenho esquemático (a) Wilhelmy Plate, e (b) Universal Sorption Device (BHASIN, 2006)

zou o microcalorímetro para medir a energia livre de superfície de agregados, determinar a energia total de adesão entre agregado e ligante asfáltico à temperatura de mistura, e medir hidrofilicidade de agregados à temperatura ambiente.

Quando um sólido é imerso em um líquido, uma nova interface sólido-líquido é formada. Essa interação é associada com a mudança na energia do sistema e é chamada de entalpia de imersão. A entalpia de imersão representa a força da interação na interface sólido-líquido. $\mathrm{Na}$ ausência de reações químicas a entalpia de imersão representa a redução na energia total do sistema devido às energias livres de superfície dos dois materiais. No caso de agregado e ligante asfáltico 
porém, essa redução deve-se muitas vezes à adsorção de grupos funcionais do ligante na superfície do agregado. Uma vantagem na utilização do microcalorímetro é que o equipamento é capaz de capturar, além da redução na energia devido à nova interface sólidolíquido, a energia devido às interações químicas. A presença de aditivos, modificadores, ou mesmo agregados ativos, torna necessária a consideração da energia adicional gerada por essas interações, que não são consideradas por meio da Equação 1.

Neste trabalho, utilizou-se um microcalorímetro diferencial isotérmico produzido pela Omnical Inc. (Figura 4(a)) para medir a entalpia de imersão quando soluções asfálticas produzidas com tolueno foram postas em contato com agregado, conforme ilustrado no esquema da Figura 4(b). O calor liberado é chamado de energia total de adesão.

\subsection{Materiais}

Neste experimento, os agregados foram separados passando na peneira \#100 e retidos na peneira \#200. Estes são lavados utilizando água destilada corrente, colocados em estufa por um período de 12 a $16 \mathrm{~h}$ à $150^{\circ} \mathrm{C}$, em seqüência deixados até que atinjam temperatura ambiente, e por fim colocados em recipientes fechados. É importante garantir que materiais maiores que a peneira $\# 100$ e inferiores à peneira \#200 sejam removidos por peneiramento e durante a lavagem, visto que, por terem área superficial diferentes, causarão erro nas medidas obtidas através do microcalorímetro.

Os ligantes asfálticos foram utilizados na forma de solução em tolueno, à temperatura ambiente. O procedimento de preparação da solução consta da dissolução de $1,5 \mathrm{~g}$ de asfalto em $11,0 \mathrm{~mL}$ de tolueno HPLC (high-performance liquid chromatography). As soluções após preparadas são deixadas por um período de $12 \mathrm{a} 24 \mathrm{~h}$ nos recipientes descritos no item a seguir, de forma que o ligante seja naturalmente dissolvido, sem necessidade de agitação. A vantagem na utilização de solução em substituição ao ligante puro está nos se- guintes aspectos: (i) possibilidade de teste à temperatura ambiente, (ii) redução na variabilidade, (iii) aumento na velocidade de execução do teste, e (iv) aumento da vida útil do equipamento.

\subsection{Preparação da amostra}

Os agregados são condicionados em recipientes de vidro de $16 \mathrm{~mL}$. O mesmo tipo de recipiente é utilizado nas células de reação e de referência do microcalorímetro. O recipiente deve possuir uma tampa vazada de polipropileno com um tampão de silicone a fim de evitar a passagem de ar, e permitir também que a agulha da seringa consiga injetar a solução durante o teste. Em um dos recipientes são adicionados $8 \mathrm{~g}$ de agregado (o outro permanecendo vazio) e em seguida os dois são submetidos ao condicionamento, que consta de aquecimento de $150^{\circ} \mathrm{C}$ a um vácuo de menos de 300 millitorr durante 3 horas. Após o condicionamento, os dois recipientes devem ser esfriados naturalmente até atingir temperatura ambiente.

\subsection{Teste}

O recipiente com a amostra é colocado na célula de reação, enquanto o recipiente vazio é posto na célula de referência. Quatro seringas de $2 \mathrm{~mL}$ de capacidade são preenchidas com solução de asfalto, duas sendo posicionadas na parte superior do recipiente de reação e duas no topo do recipiente de referência. Após equilíbrio térmico, a solução de asfalto contida dentro das seringas é injetada simultaneamente dentro dos dois recipientes através dos tampões de silicone, de forma que a diferença de calor liberado entre as duas células é capturada pelo equipamento. Após injeção da solução, as células são novamente deixadas em repouso dentro do equipamento até que o equilíbrio térmico seja novamente atingido. Os $4 \mathrm{~mL}$ de solução injetados na célula de reação contendo $8 \mathrm{~g}$ de agregado equivale a um percentual em massa de $6,4 \%$ de ligante asfáltico. A Figura 5 ilustra os passos descritos nos ítens 3.1 a 3.3 .

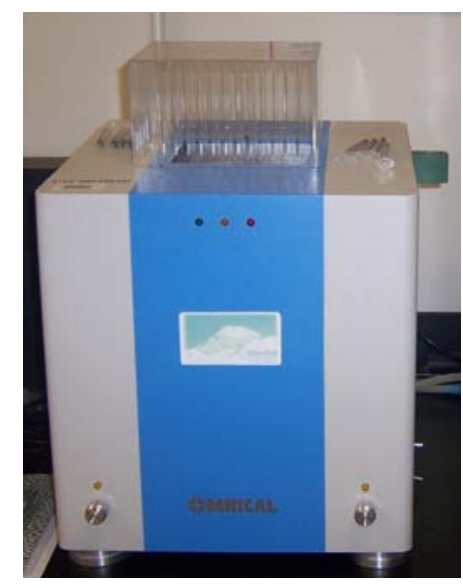

(a)

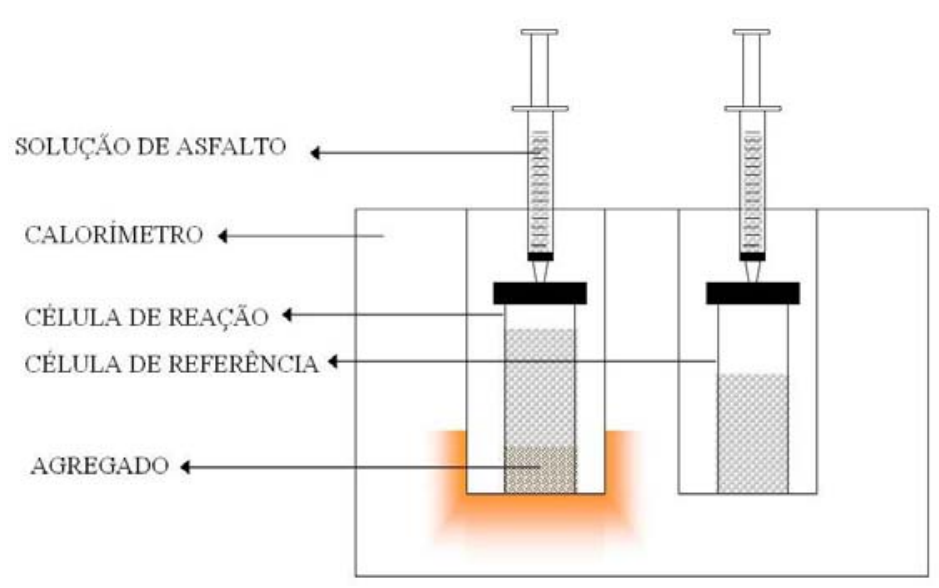

(b)

Fiqura 4: Microcalorímetro 


\subsection{Análise dos resultados}

O fluxo de calor existente entre a célula de reação e a célula de referência é determinado através de um sotware que acompanha o microcalorímetro. Sobre condições ideiais, o fluxo entre as duas células deve ser zero, porém devido à diferença de calor específico da amostra e do recipiente, além de outras condições de ensaio, uma pequena quantidade pode ser observada antes da injeção da solução no sistema. Quandos as células estão em equilíbrio térmico, esse fluxo passa a ser constante e pode ser facilmente identificado por uma linha horizontal no gráfico de fluxo de calor versus tempo (Figura 6). O calor de imersão dos agregados na solução é determinada através da integração da curva de fluxo de calor entre dois pontos de equilíbrio térmico, que indica a total energia de adesão entre o agregado e a solução de asfalto.

É importante mencionar que o calor diferencial entre as duas células medido pelo microcalorímetro de- ve-se à: (i) entalpia de imersão na célula de reação $\left(\Delta H_{i m m}\right)$, e (ii) diferença no calor de vaporização do tolueno devido a diferença do volume livre entre as duas células $\left(\Delta H_{\delta v}\right)$. Dessa forma, a entalpia de imersão é determinada através da Equação 3:

$$
\begin{aligned}
& \Delta H_{\text {imm }}=\Delta H_{\text {medido }}-\Delta H_{\delta v} \\
& \Delta H_{\text {imm }}=\Delta H_{\text {medido }}-\Delta H_{\text {vap }} \frac{v_{s} p_{o}}{R T}
\end{aligned}
$$

sendo,

$\Delta H_{\text {vap }}$ mudança de entalpia devido ao calor de vaporização por mol de tolueno,

$v_{s} \quad$ o volume ocupado pelo agregado dentro da célula de reação,

$p_{o} \quad$ a pressão de vapor de saturação do tolueno na temperatura de teste,

$R \quad$ a constante universal dos gases, $\mathrm{e}$

$T \quad$ a temperatura de teste.

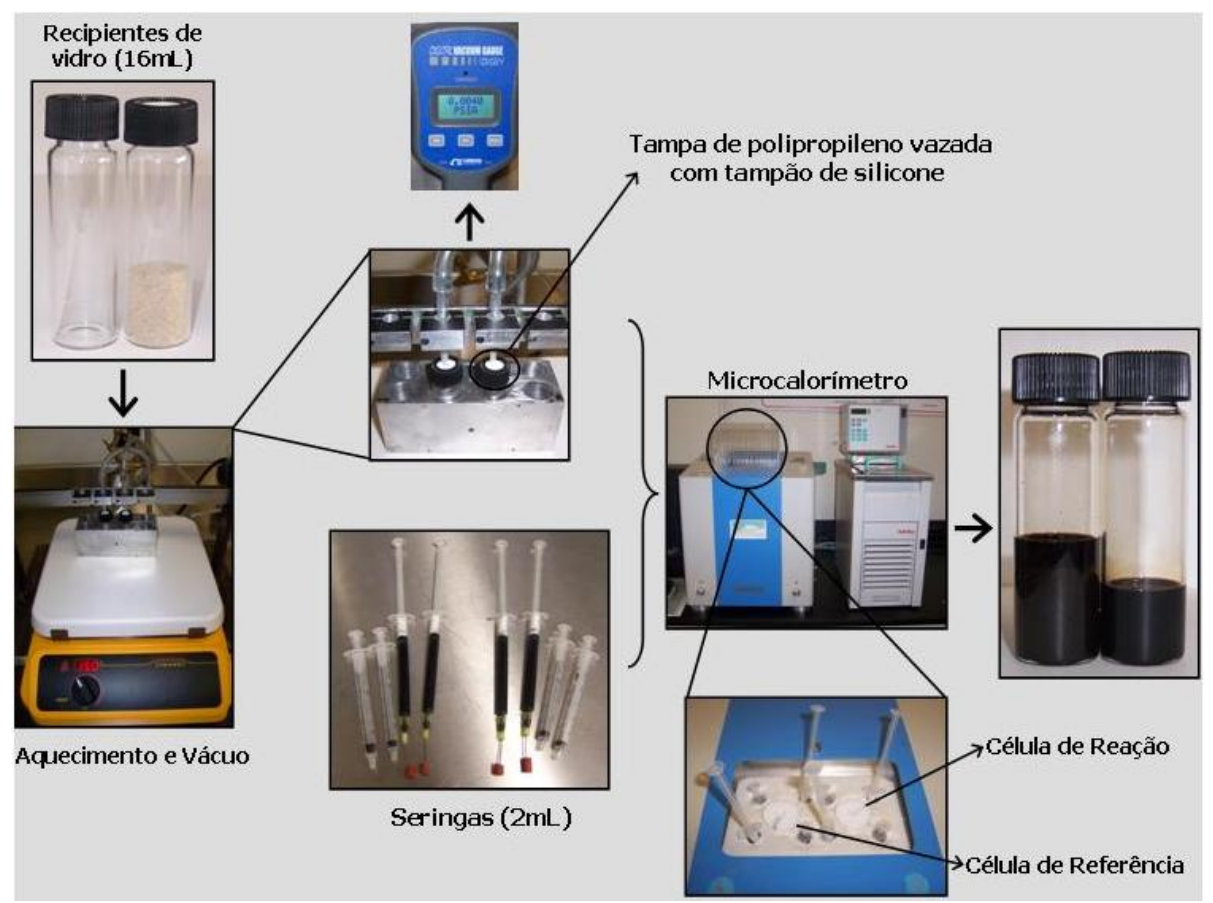

Figura 5: Resumo dos passos seguidos na preparação da amostra e teste no microcalorímetro

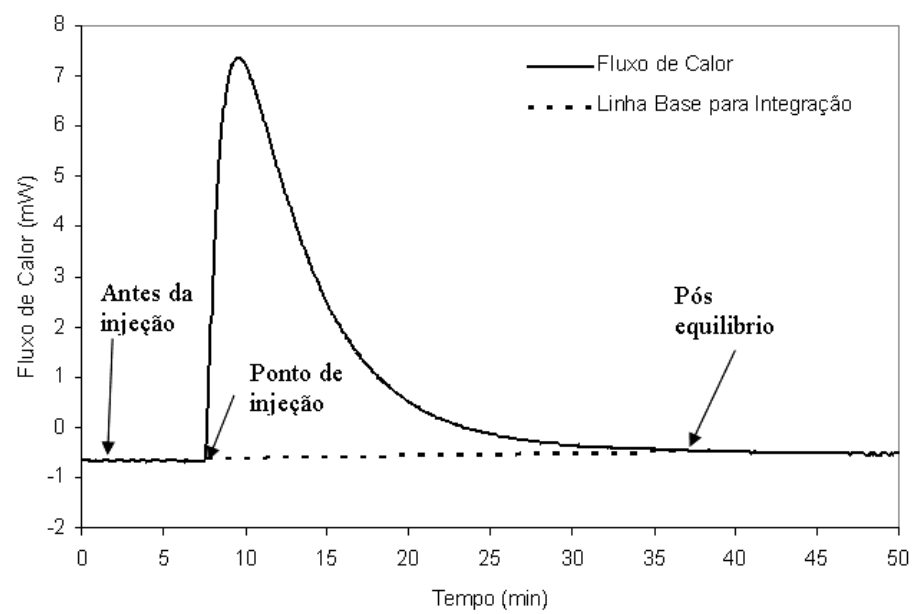

Figura 6: Curva de fluxo de calor padrão obtida através do microcalorímetro 


\section{MATERIAIS UTILIZADOS}

Agregado e ligantes asfálticos foram selecionados da biblioteca de referência do SHRP. De acordo com a nomenclatura adotada pelo SHRP foram utilizados: agregado RL, e asfaltos AAB, AAD, e ABD. O agregado RL é um pedregulho de coloração amarelada e composição variada, conforme ilustrado no gráfico da Figura 7. Os asfaltos encontram-se brevemente descritos na Tabela 1. Maiores informações sobre os materiais podem ser encontradas em Robi et al. (1991) e Jones IV (1993).

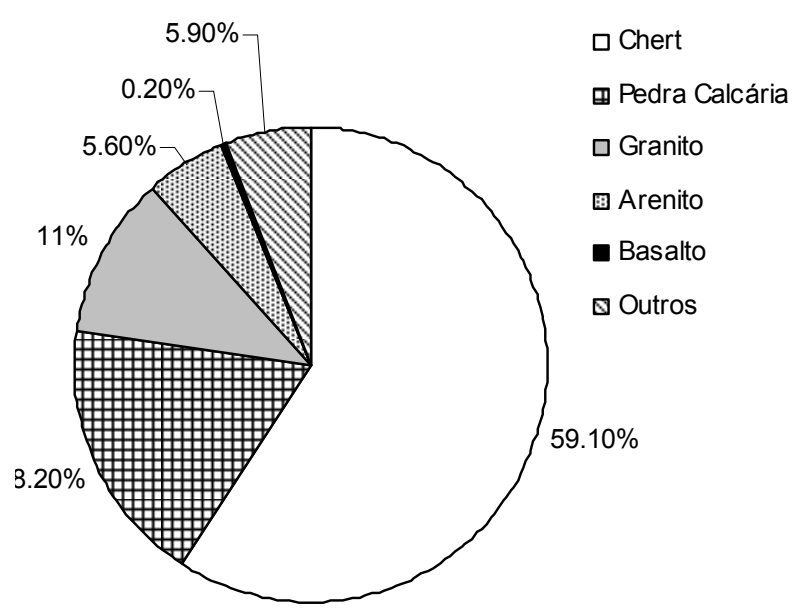

Figura 7: Composição litológica do agregado RL (ROBI et al., 1991)

\section{RESULTADOS E DISCUSSÃO}

Conforme mencionado no item 2.1, os componentes da energia de superfície do agregado (RL), e dos asfaltos (AAB, AAD e ABD) foram determinados através do WP e USD, respectivamente. Os resultados mostram que a energia livre de superfície do agregado é dominada pelo componente Lewis básico (Tabela 2), enquanto os ligantes asfálticos pelo componente nãopolar (Lifshitz-van der Waals).

Após a determinação dos componentes da energia livre de superfície dos materiais, determinou-se o trabalho de adesão entre agregado e ligante através da Equação 1. Esses valores foram comparados com os resultados da entalpia de imersão obtida através do microcalorímetro. Os resultados encontram-se apresentados na Tabela 3 e na Figura 8. Vale salientar que a unidade de medida de energia livre de superfície, bem como do trabalho de adesão, e da entalpia de imersão é reportada em unidade de energia, por unidade de área, nesse trabalho ergs $/ \mathrm{cm}^{2}$.

De acordo com os resultados mostrados, observouse um aumento na adesão das combinações RLAAD e RLABD quando utilizou-se o microcalorímetro. Esse aumento pode ser explicado por possíveis interações químicas entre agregado e ligante asfáltico, que não são capturadas através do cálculo do trabalho de adesão. Optou-se por avaliar a presença de alguns grupos funcionais nos ligantes estudados. A Tabela 4 apresenta os resultados da análise de infravermelho realizada pela Western Research Institute (WRI) nos asfaltos utilizados nessa pesquisa. Na tabela estão dispostos apenas quatro grupos funcionais apontados por Plancher et al. (1977) e Petersen et al. (1982) como um dos grupos mais fortemente adsorvidos pelas superfícies dos agregados. O asfalto AAD possui quantidade mais elevada desses grupos funcionais quando comparado com os asfaltos $\mathrm{AAB}$ e $\mathrm{ABD}$, podendo ser uma das causas da maior entalpia de imersão.

As diferenças entre os dois processos para as combinações RL AAB e RL ABD não puderam ser consideradas significativas. Os coeficientes de variação (CV) para os testes no microcalorímetro variaram de 7

Tabela 1: Características dos asfaltos AAB, AAD e ABD (JONES IV, 1993)

\begin{tabular}{llcc}
\hline Características & AAB & AAD & ABD \\
\hline & Asfalto Virgem & & $58-10$ \\
\hline PG (Performance Grade) & $58-22$ & $58-28$ & 2112 \\
Viscosidade & & & 241 \\
$140^{\circ} \mathrm{F}\left(60^{\circ} \mathrm{C}\right)$, Poise & 1029 & 309 & 47 \\
$275^{\circ} \mathrm{F}\left(135^{\circ} \mathrm{C}\right), \mathrm{cSt}$ & 289 & 135 & 1 \\
Penetração & & 9 & -0.1208 \\
$77^{\circ} \mathrm{F}\left(25^{\circ} \mathrm{C}\right), 100 \mathrm{~g}, 5 \mathrm{~s}$ & & 3800 \\
$39.2^{\circ} \mathrm{F}\left(4^{\circ} \mathrm{C}\right), 100 \mathrm{~g}, 5 \mathrm{~s}$ & 291 & -0.8102 & 321 \\
\hline
\end{tabular}

Tabela 2: Energia livre de superfície dos materiais $\left(\mathrm{ergs} / \mathrm{cm}^{2}\right)$

\begin{tabular}{lccc}
\hline Materiais & $\gamma^{L W}$ & $\gamma^{+}$ & $\gamma^{-}$ \\
\hline RL & 57,50 & 23,00 & 973,00 \\
\hline AAB & 13,58 & 2,68 & 0,00 \\
AAD & 18,47 & 0,06 & 0,10 \\
ABD & 32,45 & 0,40 & 0,00 \\
\hline
\end{tabular}


a 9\%. Com relação ao trabalho de adesão obtido pela Equação 1, os coeficientes de variação são provenientes dos testes realizados no WP e no USD. O WP apresenta valores de CV entre 2 e $3 \%$, enquanto o USD entre 10 e $15 \%$.

Tabela 3: Medidas de adesão entre agregado e ligantes asfálticos

\begin{tabular}{lcc}
\hline $\begin{array}{l}\text { Agregado/ } \\
\text { Ligante asfáltico }\end{array}$ & $\begin{array}{c}\text { Trabalho de } \\
\text { adesão }- \text { Equa- } \\
\text { ção } 1\left(\mathrm{ergs} / \mathrm{cm}^{2}\right)\end{array}$ & $\begin{array}{c}\text { Entalpia de imersão - } \\
\text { Microcalorímetro } \\
\left(\mathrm{ergs} / \mathrm{cm}^{2}\right)\end{array}$ \\
\hline RL AAB & 158 & 137 \\
RL AAD & 83 & 162 \\
RL ABD & 126 & 144 \\
\hline
\end{tabular}

Tabela 4: Grupos funcionais nos ligantes asfálticos (Jones IV, 1993)

\begin{tabular}{llll}
\hline Analise dos Grupos Funcionais & AAB & AAD & ABD \\
\hline Ácido Carboxílico & $<0.005$ & 0.015 & 0.025 \\
Quinolones & 0.012 & 0.027 & 0.015 \\
Fenóis & 0.060 & 0.130 & 0.077 \\
Anidrido $^{*}$ & 0.000 & 0.000 & 0.000 \\
\hline
\end{tabular}

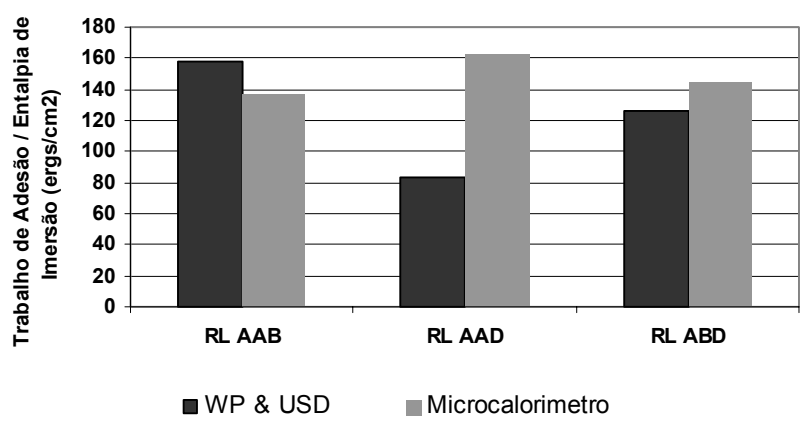

Figura 8: Medidas de adesão entre agregado e ligantes asfálti$\cos$

Especulando um pouco mais sobre o motivo da maior diferença entre trabalho de adesão e calor de imersão para a combinação RL AAD, observou-se que esse asfalto apresenta a maior mudança percentual nas viscosidades após o envelhecimento pelo TFOT (Tabela 1). Na Tabela 4, valores nulos foram reportados para o grupo funcional anidrido em todos os ligantes, o que era esperado visto que estes foram testados virgens. Porém, durante o processo de confecção das soluções, ou mesmo durante o teste, o asfalto AAD pode ter passado por um processo de envelhecimento precoce por oxidação, causando o aparecimento do anidrido em sua composição. O fato do anidrido estar entre os grupos funcionais mais fortemente adsorvidos pela superfície dos agregados, pode ter levado à maior diferença mencionada no começo do parágrafo. Estudos vêm sendo realizados a fim obter mais informações sobre o processo de envelhecimento das soluções de asfalto, bem como sua possível influência nos valores de entalpia de imersão. Bhasin et al. (2007) avaliaram o efeito do envelhecimento de ligantes asfálticos na determinação de sua energia livre de superfície, e concluíram que os resultados estão relacionados com a quantidade de grupos funcionais polares presentes no ligante.

\section{CONCLUSÕES}

Os resultados obtidos com o microcalorímetro mostraram que o equipamento possui capacidade para detectar o efeito da composição dos ligantes asfálticos nas medidas de adesão entre agregados e ligante. $\mathrm{O}$ equipamento é sensível à ocorrência de interações químicas entre os materiais envolvidos no processo, de forma que o resultado pode ser também considerado a energia total de adesão entre agregado e ligante asfáltico. $\mathrm{O}$ teste mostrou-se rápido, de fácil execução, e boa repetibilidade. Outros estudos vêm sendo realizados com o microcalorímetro na Texas A\&M, contando com a utilização de outros agregados e variações nas condições de ensaio. O objetivo é avaliar a sensibilidade do teste a mudanças de temperatura, e à preparação da solução de asfalto.

\section{REFERÊNCIAS BIBLIOGRÁFICAS}

Allen, K.W. (1992) Mechanical Theory of Adhesion. Handbook of Adhesion, p. 273-275

Bhasin, A. (2006) Development of Methods to Quantify BitumenAggregate Adhesion and Loss of Adhesion due to Water. Tese de Doutorado, Texas A\&M University, USA.

Bhasin, A.; Howson, J.; Masad, E.; Little, D.N.; E Lytton, R.L. (2007) Effect of Modification Processes on Bond Energy of Asphalt Binders. $86^{\text {th }}$ Annual Meeting, Transportation Research Board, Washington, D.C.

Cheng, D.; Little, D.N.; Lytton, R.L.; e Holste, J.C. (2002a) Surface Energy Measurements of Asphalt and its Application to Predicting Fatigue and Healing in Asphalt Mixtures. $81^{\text {st }}$ Annual Meeting, Transportation Research Board, Washington, D.C

Cheng, D. (2002b) Surface Free Energy of Asphalt-Aggregate System and Performance Analysis of Asphalt Concrete Based on Surface Free Energy. Tese de Doutorado, Texas A\&M University.

Curtis, C.W.; Terrel, R.L.; Perry, L.M.; Al-Swailm, S.; e Braanan, C.J. (1991) Importance of Asphalt-Aggregate Interactions in Adhesion. Journal of the Association of Asphalt Paving Technologists, v. 60, p. $476-532$

Dessouky, S.; Masad, E.; e Little, D. (2006) Mechanistic Modeling of Permanent Deformation in Asphalt Mixes with the Effect of Aggregate Characteristics. Journal of the Association of Asphalt Paving Technologists, v. 75, p. 535-571.

Ensley, E.K.; Petersen, J.C.; e Robertson, R.E. (1984) Asphalt-Aggregate Bonding Energy Measurements by Microcalorimetric Methods. Thermochimica Acta, v. 77, p. 95-107.

Hefer, A.W.; Little, D.N.; e Lytton, R.L. (2005) A Synthesis of Theories and Mechanisms of Bitumen-Aggregate Adhesion Including Recent Advances in Quantifying the Effects of Water. Journal of the Association of Asphalt Paving Technologists, v. 74, p. 139-196.

Hefer, A.W.; Bhasin, A.; e Little, D.N. (2006) Bitumen Surface Energy Characterization Using a Contact Angle Approach. Journal of Materials in Civil Engineering, ASCE, v. 18, No. 6, December 1, p. 759-767.

Jeon, Y.W. e Curtis, C.W. (1990) A Literature Review of the Adsorption of Asphalt Functionalities on Aggregate Surfaces. Strategic Highway Research Program, Report SHRP-A/IR-90-014, Washington, D.C., National Research Council.

Jones IV, D.R. (1993) SHRP Materials Reference Library: Asphalt Cements : A Consice Data Compilation, SHRP-A-645, Contract A001, Strategic Highway Research Program, National Research Council, Washington, D.C.

Little, D.N. e Petersen, C. (2005) Unique Effects of Hydrated Lime Filler on the Performance-Related Properties of Asphalt Cements 
Physical and Chemical Interactions Revised. Journal of Materials in Civil Engineering, ASCE, v. 17, No. 2, April 1, 2005.

Lytton, R.L. (2004) Adhesive Fracture in Asphalt Concrete Mixtures. Capítulo de Livro Editado por J. Youtcheff, Submetido para Publicação.

Masad, E.; Zollinger, C.; Bulut, R.; Little, D.N.; e Lytton, R.L. (2006) Characterization of HMA Moisture Damage Using Surface Energy and Fracture Properties. Journal of the Association of Asphalt Paving Technologists, v. 75, p. 713-754.

Marek, C.R.; e Herrin, M. (1958). Tensile Behavior and Failure of Thin Films of Asphalt. Journal of the Association of Asphalt Paving Technologists, v. 37, 386-421.

Petersen, J.C.; Plancher, H.; Ensley, E.K.; Miyake, G., e Venable, R.L. (1982) Chemistry of Asphalt-Aggregate Interaction: Relationship with Moisture Damage Prediction Test. Transportation Research Record, 843, p. 95-104.

Plancher, H.; Dorrence, S.M.; e Petersen, J.C. (1977) Identification of Chemical Types in Asphalt Strongly Adsorbed at the AsphaltAggregate Interface and Their Relative Displacement by Water. Journal of the Association of Asphalt Paving Technologists, v. 46, p. $151-175$.

Robert, A.D. (1977) Surface Charge Contribution in Rubber Adhesion and Friction. Journal of Physics D: Applied Physics, v. 10, p. 18011819.

Robi, T.L.; Milburn, D.; Thomas, G.; Groppo, J.; O'hara, K.; e Haak, A. (1991) The SHRP Materials Reference Library Aggregates: Chemical, Mineralogical, and Sorption Analysis, SHRP-A/UIR91-509, Contract A-001, Strategic Highway Research Program, National Research Council, Washington, D.C.

Tarrar, A.R. e Wagh, V.P. (1992) The Effect of the Physical and Chemical Characteristics of the Aggregate on Bonding. Strategic Highway Research Program, Report SHRP-A/UIR-91-507, Washington, D.C., National Research Council.

Van Oss, C.J.; Chaudhury, M.K.; e Good, R.J. (1988) Interfacial Lifshitzvan der Waals and Polar Interactions in Macroscopic Systems. Chemical Reviews, v. 88, p. 927-941.

Van Oss, C.J. (1994) Interfacial Forces in Aqueous Media, Marcel Dekler, Inc., NY. 